\title{
Applying Machine Learning Techniques to Identify Undiagnosed Patients with Exocrine Pancreatic Insufficiency
}

\author{
Bruce Pyenson ${ }^{1}$, Maggie Alston $^{1 *}$, Jeffrey Gomberg ${ }^{2}$, Feng Han ${ }^{3}$, Nikhil Khandelwal ${ }^{4}$, Motoharu Dei $^{3}$, \\ Monica Son ${ }^{3}$ Jaime Vora ${ }^{4}$ \\ ${ }^{1}$ Milliman, New York, NY \\ ${ }^{2}$ AIBrainTree, LLC, Chicago, IL \\ ${ }^{3}$ Milliman, New York, NY, during study \\ ${ }^{4}$ AbbVie Inc, North Chicago, IL \\ *Corresponding author: maggie.alston@milliman.com
}

\begin{abstract}
Background: Exocrine pancreatic insufficiency (EPI) is a serious condition characterized by a lack of functional exocrine pancreatic enzymes and the resultant inability to properly digest nutrients. EPI can be caused by a variety of disorders, including chronic pancreatitis, pancreatic cancer, and celiac disease. EPI remains underdiagnosed because of the nonspecific nature of clinical symptoms, lack of an ideal diagnostic test, and the inability to easily identify affected patients using administrative claims data.
\end{abstract}

Objectives: To develop a machine learning model that identifies patients in a commercial medical claims database who likely have EPI but are undiagnosed.

Methods: A machine learning algorithm was developed in Scikit-learn, a Python module. The study population, selected from the 2014 Truven MarketScan ${ }^{\circledR}$ Commercial Claims Database, consisted of patients with EPI-prone conditions. Patients were labeled with 290 condition category flags and split into actual positive EPI cases, actual negative EPI cases, and unlabeled cases. The study population was then randomly divided into a training subset and a testing subset. The training subset was used to determine the performance metrics of 27 models and to select the highest performing model, and the testing subset was used to evaluate performance of the best machine learning model.

Results: The study population consisted of 2088 actual positive EPI cases, 1077 actual negative EPI cases, and 437530 unlabeled cases. In the best performing model, the precision, recall, and accuracy were $0.91,0.80$, and 0.86 , respectively. The best-performing model estimated that the number of patients likely to have EPI was about 12 times the number of patients directly identified as EPI-positive through a claims analysis in the study population. The most important features in assigning EPI probability were the presence or absence of diagnosis codes related to pancreatic and digestive conditions.

Conclusions: Machine learning techniques demonstrated high predictive power in identifying patients with EPI and could facilitate an enhanced understanding of its etiology and help to identify patients for possible diagnosis and treatment.

Keywords: machine learning, case-finding technique, claims data analysis, exocrine pancreatic insufficiency (EPI), identifying/ predicting undiagnosed EPI, predictive modeling 


\section{Background}

Exocrine pancreatic insufficiency (EPI) is a serious condition characterized by a lack of functional exocrine pancreatic enzymes and the resultant inability to properly digest fats, carbohydrates, and proteins. ${ }^{1}$ Common pancreatic causes of EPI include chronic pancreatitis, severe acute pancreatitis, pancreatic cancer, pancreatic surgery, and cystic fibrosis. ${ }^{1-3}$ Non-pancreatic causes of EPI include a history of celiac disease, diabetes mellitus, Crohn's disease, gastric surgery, short bowel syndrome, and Zollinger-Ellison syndrome. ${ }^{1,3}$ The main symptoms of EPI are steatorrhea (ie, excess fat in the stool), abdominal bloating/discomfort, and weight loss. ${ }^{1}$

Although an early and accurate diagnosis of EPI is critically important to optimize patient outcomes, ${ }^{3,4}$ the condition remains underdiagnosed. ${ }^{1}$ In addition, there is a lack of consensus regarding the best diagnostic approach, and experts have noted the non-reliability and non-specificity of available diagnostic tests. ${ }^{1}$ Currently available diagnostic tests for EPI include fecal fat quantification, the fecal elastase- 1 test, and the ${ }^{13} \mathrm{C}$-mixed triglyceride breath test. ${ }^{4}$ Diagnosing EPI is challenging because its symptoms may be vague or overlap with those of other gastrointestinal disorders. ${ }^{1}$ Other barriers to the diagnosis of EPI have also been identified: (1) currently available tests are cumbersome and unpleasant; ${ }^{5}$ (2) tests are not widely available or accurate for patients in the early to moderate stages of EPI; ${ }^{5}$ (3) there is no International Classification of Diseases, Ninth Revision, Clinical Modification (ICD-9-CM) or Current Procedural Terminology (CPT) code that definitively identifies a patient with EPI, thus tracking has been difficult from a claims perspective; ${ }^{6}$ and (4) use of an International Classification of Diseases, Tenth Revision (ICD-10) code introduced for EPI in October 2016 may not yet be integrated into administrative claims data or electronic medical records (EMR).

The precise incidence and prevalence of EPI are difficult to determine due to its underdiagnosis; furthermore, medical statistics on EPI are usually not reported. ${ }^{1}$ Some data suggest, however, that the prevalence of EPI in patients with chronic pancreatitis is 30\% to $40 \%$ and that the prevalence of EPI in patients with cystic fibrosis is $80 \%$ to $90 \%$. $^{2}$ Studies have also shown that more than $40 \%$ of patients with type 1 diabetes mellitus and $30 \%$ of patients with type 2 diabetes develop mild to moderate EPI. ${ }^{2}$ At present, reliable estimates of EPI prevalence in the general population are lacking. ${ }^{4}$

Left untreated, EPI can have a deleterious effect on quality of life and may lead to the development of nutritional deficiencies and subsequent malnutrition-related conditions. ${ }^{4}$ The goals of EPI treatment are to alleviate the unpleasant clinical symptoms related to maldigestion and to correct nutritional deficiencies. ${ }^{7}$ Currently, EPI is treated with lifestyle modifications (ie, smoking cessation; alcohol abstinence; and the consumption of frequent, low-volume meals) and oral pancreatic enzyme replacement therapy (PERT), the latter of which is the cornerstone of EPI treatment. ${ }^{4}$ Guidelines established by international societies are largely in agreement that more aggressive treatment of EPI is needed. ${ }^{5,8-12}$

The purpose of the current study was to identify patients in a commercial medical claims database who likely had EPI but were undiagnosed. To that end, we performed a claims data analysis using machine learning, a data analytics approach that is being increasingly used as a predictive tool in the field of medicine. ${ }^{13-24}$

\section{Methods}

\section{Data Source}

We used the 2014 Truven MarketScan ${ }^{\circledR}$ Commercial Claims Database (hereafter MarketScan) to identify the study population. MarketScan is a large dataset that contains enrollment data and health benefit claims data for more than 39 million commercially insured lives from more than 100 employer and health plan contributors. 
The enrollment data include beneficiary age, dependent status, and monthly eligibility status. The claims data include coding information (diagnosis, procedure, revenue, diagnosis-related group, and national drug codes), service dates, and site of service.

\section{Study Population}

The source population consisted of commercially insured individuals selected from the MarketScan database who were 0-64 years old at the end of 2014 and were active or disabled (not part-time or temporary) employees or their dependents. In addition, individuals were required to have prescription drug coverage for the entire duration of their medical coverage period and could not be enrolled in capitated health plans.

For modeling, we extracted all claims from the source data and constructed an enriched study population by selecting individuals who fell into broad categories of diagnoses that are prone to EPI. These categories included inflammatory conditions of the pancreas, other pancreatic conditions (including unspecified diseases of the pancreas), malabsorption syndromes, inflammatory bowel disease, insulin-taking diabetes, and HIV; individuals who had undergone bariatric surgery were also included. We excluded individuals who had conditions that were almost certainly associated with EPI (ie, cystic fibrosis and pancreatic cancer) and those who had undergone radical pancreatic surgeries (ie, pancreaticoduodenectomy, radical subtotal pancreatectomy, or total pancreatectomy). It is highly likely that the excluded individuals would be recognized as being at high risk of EPI, thus we did not want their characteristics to overshadow the characteristics of less EPI-prone cohorts. Non-insulin-dependent diabetics were also excluded from the study population after preliminary data analyses found a low association of EPI in that group. See Table A1 in the Technical Appendix for a full list of the conditions used to include and exclude patients from the study population.

\section{Machine Learning Algorithm}

Machine learning is a computer-based data analytics approach that automates model building through the use of algorithms that iteratively learn from and adapt to subject data. ${ }^{25}$ To develop our models, we used the open source library Scikit-learn, a Python module featuring machine learning algorithms. ${ }^{26}$

Machine learning uses terminology that differs from that of statistical analysis, the latter of which may be more familiar to the health care readership. A basic knowledge of machine learning terminology, however, is needed to understand the methodology used in our analysis. See Table 1 for a comparison of the terminologies used in machine learning and statistical analysis.

Machine learning often uses highly technical iterative processes and trials to develop workable models. A common practice in machine learning is to explore several approaches to improve model fit.

We used a three-step procedure to create the main input to our machine learning model.

1) Two hundred and ninety condition category flags (features) were constructed. These categories were composed of broad, clinically-related ranges of various codes: 74 ICD-9-CM diagnosis code categories, 5 ICD-9-CM procedure code categories, 37 CPT code categories, 70 revenue code (REVCODE) categories, and 104 national drug code (NDC) categories.

2) Each claim for each member of the study population was assigned a flag for each condition category to indicate whether the condition was present $(\mathrm{flag}=1)$ or not present $(\mathrm{flag}=0)$.

3) Claim-level flags were summarized to create a single set of the 290 condition category flags for each member in the study population. The value in each condition category indicated the total number of claims in that category. Patient age and sex, as well as prescription counts for patients who had been 
treated with a PERT, were also used to create the machine learning model.

We used a nine-step procedure to create our machine learning model and to generate, validate, and assess the results.

1) The study population was divided into three groups: actual positive cases (patients believed to have EPI), actual negative cases (patients believed to not have EPI), and unlabeled cases (patients with unknown EPI status). Actual positive cases (or "true positives") included patients who had filled $\geq 3$ prescriptions for a PERT. Actual negative cases (or "true negatives") included patients who had undergone a fecal elastase-1 test (CPT code 82656) but had not filled a PERT prescription.

2) The study population was randomly divided into an $80 \%$ training subset and a $20 \%$ testing subset. The training subset was used to determine the performance metrics and select the highest performing model, and the testing subset was used to evaluate performance of the best-performing machine learning model using data not used to train or select the model.

3) A baseline model was created to predict the classification of an observation based on several input variables - in this case, to classify whether a patient has or does not have EPI. The latter was accomplished using least absolute shrinkage and selection operator (LASSO) with logistic regression, a method that is commonly used to classify data. ${ }^{27}$ LASSO penalizes the absolute size of the regression coefficients, which in practice, leads to sparse coefficients of the input variables in the logistic regression and is helpful when there are many correlated input variables.

4) Additional models were created using other machine learning techniques with different underlying frameworks to determine which framework was best for the input data and model goals. The additional machine learning techniques included gradient-boosted classification trees, support vector machines, and random forest. Random forest is an ensemble method that leverages a collection of decision trees that generate a response when presented with a set of features.

5) Adjustments were applied to handle "imbalanced data," which occurs when one or more of the binary classes is underrepresented, and included modifying class weights, oversampling, and undersampling. See Table A2 in the Technical Appendix.

6) A $3 \times 3$ nested cross validation process was applied to several random forest models to determine the hyperparameters (ie, the set of parameters that defines the properties of the model, such as the number of decision trees in a random forest) and to generate performance metrics. This process was applied to the training subset and consisted of two steps: outer cross validation and inner cross validation. In the outer cross validation step, the training subset was split into three folds (groups). Two of the folds were used to train the model on the parameters, and the remaining fold acted as the validation set. This process was repeated until every fold acted as the validation set one time. Within the training folds assigned by the outer cross validation step, the folds were further split into three sub-folds for the inner cross validation process. Two of the sub-folds were used to tune the parameters, and the remaining sub-fold acted as the validation set. The hyperparameters were optimized by selecting the best performing set, as measured by the average performance metrics over three validation sets through inner cross validation. The performance metrics of the selected random forest models were then calculated in the validation set through outer cross validation. The inner cross validation process was repeated separately over the three outer cross validation splits; that is, we had three sets of hyperparameters from three inner loops and three sets of performance metrics from three outer loops. The performance metrics were averaged for the purpose of choosing the best-performing model. ${ }^{28}$ See Tables A3 and A4 in the Technical Appendix.

7) The performance metrics of 27 models were examined. Model performance was evaluated using each patient's outputted EPI probability. The metrics used to optimize hyperparameters or to compare the baseline model with other models included precision, recall, $\mathrm{F}_{1}$ score, $\mathrm{F}_{\text {beta }=10}$ score, positive-unlabeled (PU) score, and Brier score loss; see Table 2.

8) The best-performing model was retrained on the full $80 \%$ training subset and applied to the $20 \%$ 
testing subset to calculate the performance metrics. Results were summarized in a confusion matrix, which is a commonly used template for presenting the results of binary classifications in machine learning. A receiver-operator characteristic curve was developed.

9) After assigning the probability of EPI, we further examined patients' claims to determine whether the probability assignments were clinically reasonable. To that end, we used two approaches. In the first approach, we extracted cohorts of patients in the $10 \%$ to $20 \%$ EPI probability bucket and the $80 \%$ to 90\% EPI probability bucket and summarized the top 30 code counts for patients in each bucket. In the second approach, we generated a relative importance measure for each feature based on its unique impact on the overall Gini impurity of the model. Gini impurity is a measure of the randomness of the model being evaluated, and the sum of the relative Gini impurity measures for all features is 1.00. Our goal was to minimize the Gini impurity of the model in order to optimize the accuracy of patient classification.

\begin{tabular}{ll}
\hline Table 1. Terminology Used in Machine Learning and Statistical Analysis \\
\hline Machine Learning & Statistical Analysis \\
\hline Feature & Explanatory variable \\
Confusion matrix & Contingency table of predicted and actual status \\
Recall & Sensitivity \\
Precision & Positive predictive value \\
F1 score & Harmonic mean of sensitivity and positive predictive value \\
\hline
\end{tabular}

\begin{tabular}{ll}
\hline Table 2. Performance & Metrics Definitions \\
\hline Metric & Definition \\
\hline Precision & $\begin{array}{l}\text { Measure of model exactness; the ratio of successful model predictions over all cases } \\
\text { predicted to be positive } \\
\text { Mecall }\end{array}$ \\
$\begin{array}{l}\text { cases that are actual positives } \\
\text { Measure of model accuracy; the harmonic mean of precision and recall (reciprocal } \\
\text { of the mean of the reciprocals of precision and recall) }\end{array}$ \\
$\begin{array}{l}\text { Similar to } \mathrm{F}_{1} \text { score with an additional parameter (beta) that assigns greater weight to } \\
\mathrm{F}_{\text {beta=10 }} \text { score }\end{array}$ \\
$\begin{array}{l}\text { Positive-unlabeled score } \\
\text { Brier score loss }\end{array}$ & $\begin{array}{l}\text { Measure of model performance that is positively correlated with the } \mathrm{F}_{1} \text { score } \\
\text { Measure of the mean squared differences of the outcome and predictive probability }\end{array}$ \\
\hline
\end{tabular}

\section{Results}

In total, 440695 patients out of the 39 million patients in the MarketScan database met the study inclusion criteria. Of these patients, 2088 were actual positive EPI cases and 1077 were actual negative EPI cases. The remaining 437530 patients were unlabeled cases. Because actual positives and actual negatives were underrepresented, the data was considered imbalanced.

We found that the three models with the highest performance scores were all random forest models. The baseline LASSO model demonstrated the highest performance in recall, Brier score loss by labeled data, and $\mathrm{F}_{1}$ score; however, the baseline model was not the highest performing model overall because it generated an unreasonably high count of positive cases in the unlabeled data (0.93). Three random forest models, which assumed that unlabeled data represented negative EPI cases, generated high recall and precision on the labeled data. These models also accurately estimated a small number (between 0.04 and 0.06 ) of positive cases in the unlabeled data and a small Brier score loss (between 0.14 and 0.16) on the full dataset, thereby 
making them high-performing models. See Table 3.

Table 3. Results of Baseline and High-performing Models

\begin{tabular}{|c|c|c|c|c|}
\hline & Baseline & 1 & 2 & 3 \\
\hline Model Type & LASSO & Random Forest & Random Forest & Random Forest \\
\hline \multicolumn{5}{|l|}{ Metrics } \\
\hline Unlabeled data & $\begin{array}{l}\text { Ignored } \\
\text { unlabeled data }\end{array}$ & $\begin{array}{l}\text { Assumed negative, } \\
\text { ignored actual } \\
\text { negative cases }\end{array}$ & $\begin{array}{l}\text { Assumed negative, } \\
\text { ignored actual } \\
\text { negative cases }\end{array}$ & $\begin{array}{l}\text { Assumed } \\
\text { negative, ignored } \\
\text { actual negative } \\
\text { cases }\end{array}$ \\
\hline Imbalanced data & $\mathrm{N} / \mathrm{A}$ & $\begin{array}{l}\text { Downsample, class } \\
\text { weight }\end{array}$ & $\begin{array}{l}\text { Downsample, } \\
\text { subsample balanced } \\
\text { weight }\end{array}$ & $\begin{array}{l}\text { Repeated random } \\
\text { subsampling }\end{array}$ \\
\hline Validation method & $\begin{array}{l}80 \% / 20 \% \text { split } \\
\text { validation }\end{array}$ & $\begin{array}{l}\text { Nested cross } \\
\text { validation }\end{array}$ & $\begin{array}{l}\text { Nested cross } \\
\text { validation }\end{array}$ & $\begin{array}{l}\text { Nested cross } \\
\text { validation }\end{array}$ \\
\hline $\begin{array}{l}\text { Optimized metric in } \\
\text { hyperparameter selection }\end{array}$ & None & $\begin{array}{l}\mathrm{F}_{\text {(beta }=10)} \text { using } 100 \\
\text { random iterations }\end{array}$ & $\begin{array}{l}\mathrm{F}_{(\text {beta }=10)} \times 100+ \\
\text { PU score using } 100 \\
\text { random iterations }\end{array}$ & $\begin{array}{l}\mathrm{F}_{(\text {beta }=10)} \times 100+ \\
\text { PU score using } 60 \\
\text { random iterations }\end{array}$ \\
\hline \multicolumn{5}{|l|}{ Scores } \\
\hline $\mathrm{F}_{\text {beta }=10}$ score (all data) & 0.32 & 0.71 & 0.71 & 0.72 \\
\hline PU score (all data) & 0.93 & 9.22 & 12.45 & 10.69 \\
\hline Recall (labeled data) & 0.92 & 0.81 & 0.77 & 0.80 \\
\hline Brier score loss (labeled data) & 0.10 & 0.14 & 0.16 & 0.15 \\
\hline $\begin{array}{l}\text { Brier score loss (unlabeled data } \\
\text { assumed negative) }\end{array}$ & 0.60 & 0.06 & 0.03 & 0.04 \\
\hline $\mathrm{F}_{1}$ score (labeled data) & 0.90 & 0.86 & 0.84 & 0.86 \\
\hline Precision (labeled data) & 0.88 & 0.91 & 0.93 & 0.91 \\
\hline $\begin{array}{l}\text { Probability of unlabeled cases } \\
\text { to be labeled as positive }\end{array}$ & 0.93 & 0.07 & 0.04 & 0.06 \\
\hline
\end{tabular}

For Model 3, the best performing of the four models, we determined that there were 336 true-positive patients (ie, patients who were accurately predicted to have EPI) and 183 true-negative patients (ie, patients who were accurately predicted to not have EPI), with predicted EPI defined as a patient with an EPI risk of $\geq 50 \%$. There were 32 false-positive patients (type I error); these were patients who were predicted to have EPI but did not actually have it. There were 82 false-negative patients (type II error); these were patients who had EPI but were not predicted to have it. See Table 4, which shows the confusion matrix for the 88,140 patients in the testing subset. 
Table 4. Confusion Matrix of Model -3

\begin{tabular}{llll}
\hline & & \multicolumn{2}{c}{ Predicted Condition } \\
\cline { 3 - 4 } & & No EPI & EPI \\
\hline \multirow{3}{*}{ No EPI } & 183 & 32 \\
& & (True negatives) & (False positives, type I error) \\
& \multirow{2}{*}{ EPI } & 82 & 336 \\
& Unknown & (False negatives, type II error) & (True positives) \\
& 82546 & 4961 \\
\hline
\end{tabular}

Note: The $2 \times 2$ cells shaded in gray represent the confusion matrix

EPI: exocrine pancreatic insufficiency

The number of patients known to have EPI in the testing subset (82 false negatives +336 true positives) was 418. Of the 87507 previously unlabeled patients in the testing subset, 4961 patients $(6 \%)$ were identified as being likely to have EPI, which is approximately 12 times the number of patients in the study population who were identified as being positive for EPI.

From the confusion matrix, we determined key evaluation metrics pertaining to the performance of Model 3, including precision, recall, and $F_{1}$ score. Precision, the ratio of true positives over the sum of true positives and false positives $(336 /\{32+336\})$, is a measure of exactness and was found to be 0.91 . Recall, the ratio of true positives over the sum of true positives and false negatives $(336 /\{82+336\})$, quantifies the completeness of model results and was found to be 0.80 . The $\mathrm{F}_{1}$ score, the harmonic mean of precision and recall, depicted as the reciprocal of the mean of the reciprocals of precision and recall $(\{0.91 \times 0.80\} /\{0.91+0.80\} \times 2)$, is a measure of accuracy that was found to be 0.86 , where 1.0 represents perfect accuracy. The area under the receiver-operator characteristic curve (AUROC) is a measure of the predictability of the model. An AUROC equal to 1.00 represents a model that perfectly predicts an outcome. An AUROC of 0.50 represents a model with no predictability. In our study, AUROC for the labeled data was found to be 0.94.

In the final step of our study, we applied Model 3 to the 20\% testing subset, which assigned each patient a probability of having EPI. We observed that out of a sample of 88140 patients, 5329 patients (6\%) were now captured as EPI patients, with EPI defined as a patient with an EPI risk of $\geq 50 \%$. If the EPI risk threshold was increased to $75 \%$, only 1376 patients $(2 \%)$ were captured as EPI patients. See Table 5.

In practice, Model 3 could be further applied to the training subset or to new datasets to identify potential previously unidentified patients with EPI. It is important to note, however, that model performance will vary when applying any model to new datasets that differ significantly from the 80\% training subset (eg, in terms of population demographics or other characteristics). In such situations, the results should be interpreted with caution, and if possible, model performance should be re-evaluated on the new population and data source.

From our lower (10\% to 20\%) and upper (80\% to 90\%) tail analysis for clinical reasonability, we observed that two CPT code features - Evaluation and Management (E\&M) (99201 - 99499) and Pathology (80047 - 89398) - were the forerunners in both probability buckets, where nearly all patients had at least one E\&M or Pathology claim. The finding was not unexpected, as the study population represented an enriched group of patients with pancreatic conditions that would require laboratory work and diagnostic or management evaluations. These two features, therefore, were not key predictors in determining EPI probability. We observed several other overlapping features between the 10\% and 20\% EPI probability bucket and the $80 \%$ to $90 \%$ EPI probability bucket, such as Mental (diagnoses) and Radiology - diagnostic (procedures); however, in the 80\% to 90\% EPI probability bucket, more features related to conditions of the pancreas and digestive system were found at the top of the list than was the case in the $10 \%$ to $20 \%$ EPI probability bucket. See Tables 6a and 6b. 


\begin{tabular}{lcc}
\hline \multicolumn{3}{l}{ Table 5. Probability of EPI Assigned to Each Patient by Model 3 } \\
\hline Probability of EPI & Number of Patients & Predicted to have EPI \\
\hline $0 \%-<5 \%$ & 35868 & \\
$5 \%-<10 \%$ & 18022 & \\
$10 \%-<15 \%$ & 11895 & \\
$15 \%-<20 \%$ & 7470 & \\
$20 \%-<25 \%$ & 4186 & \\
$25 \%-<30 \%$ & 2248 & \\
$30 \%-<35 \%$ & 1085 & \\
$35 \%-<40 \%$ & 711 & \\
$40 \%-<45 \%$ & 671 & \\
$45 \%-<50 \%$ & 655 & \\
$50 \%-<55 \%$ & 719 & \\
$55 \%-<60 \%$ & 666 & \\
$60 \%-<65 \%$ & 678 & \\
$65 \%-<70 \%$ & 884 & \\
$70 \%-<75 \%$ & 1006 & \\
$75 \%-<80 \%$ & 767 & \\
$80 \%-<85 \%$ & 397 & \\
$85 \%-<90 \%$ & 192 & \\
$90 \%-<95 \%$ & 20 & \\
$95 \%-100 \%$ & 0 & \\
Total & & \\
$0 \%-<25 \%$ & & \\
$25 \%-<50 \%$ & & \\
$50 \%-<75 \%$ & & \\
$75 \%-<100 \%$ & & \\
\hline EPI: exocrine pancreatic insufficiency to have hely to have EPI & \\
\hline
\end{tabular}


Table 6a. Most Frequent Features in Patients with 10\% to 20\% EPI Probability

\begin{tabular}{|c|c|c|c|}
\hline Feature & Code Type & $\begin{array}{c}\text { Portion of } \\
\text { Patients with } \geq 1 \\
\text { Observations }\end{array}$ & $\begin{array}{l}\text { Average Number of } \\
\text { Observations for } \\
\text { Patients with } \geq 1 \\
\text { Observations }\end{array}$ \\
\hline Evaluation and Management & CPT & $100 \%$ & 15.81 \\
\hline Pathology & $\mathrm{CPT}$ & $97 \%$ & 28.03 \\
\hline Medicine & $\mathrm{CPT}$ & $90 \%$ & 17.72 \\
\hline Special Encounters & DIAG & $78 \%$ & 24.07 \\
\hline Other Symptoms & DIAG & $77 \%$ & 21.96 \\
\hline Radiology & $\mathrm{CPT}$ & $74 \%$ & 5.85 \\
\hline Cardiovascular & $\mathrm{CPT}$ & $73 \%$ & 3.84 \\
\hline Metabolic & DIAG & $69 \%$ & 24.45 \\
\hline Musculoskeletal & DIAG & $64 \%$ & 24.15 \\
\hline Laboratory & REVCODE & $62 \%$ & 16.59 \\
\hline Diabetes & DIAG & $56 \%$ & 41.46 \\
\hline Hypertensive & DIAG & $56 \%$ & 23.94 \\
\hline Ulcer Drugs & NDC & $55 \%$ & 4.96 \\
\hline Other Analgesics & NDC & $55 \%$ & 4.56 \\
\hline Antibiotics & NDC & $55 \%$ & 2.22 \\
\hline Respiratory & DIAG & $54 \%$ & 14.80 \\
\hline Other Special Encounters & DIAG & $52 \%$ & 7.98 \\
\hline Insulin & NDC & $50 \%$ & 7.08 \\
\hline Genitourinary & DIAG & $49 \%$ & 25.70 \\
\hline Antihypertensives & NDC & $46 \%$ & 6.43 \\
\hline Digestive & DIAG & $45 \%$ & 15.10 \\
\hline Antihyperlipidemics & NDC & $44 \%$ & 6.39 \\
\hline Pharmacy & REVCODE & $43 \%$ & 4.48 \\
\hline Labs Vitamin Levels Test & $\mathrm{CPT}$ & $43 \%$ & 2.56 \\
\hline Anesthesia & $\mathrm{CPT}$ & $42 \%$ & 1.93 \\
\hline Mental & DIAG & $42 \%$ & 17.15 \\
\hline Esophagus & DIAG & $40 \%$ & 12.62 \\
\hline Radiology - Diagnostic & REVCODE & $40 \%$ & 2.07 \\
\hline Antidepressants & NDC & $39 \%$ & 6.96 \\
\hline Drugs Requiring Specific Identification & REVCODE & $38 \%$ & 6.54 \\
\hline \multicolumn{4}{|c|}{$\begin{array}{l}\text { CPT: current procedural terminology; DIAG: diagnosis; EPI: exocrine pancreatic insufficiency; NDC: national drug cod } \\
\text { REVCODE: revenue code } \\
\text { Note: Observations were tabulated at the claim level }\end{array}$} \\
\hline
\end{tabular}




\begin{tabular}{|c|c|c|c|}
\hline \multicolumn{4}{|c|}{ Table 6b. Most Frequent Features in Patients with $80 \%$ to $90 \%$ EPI Probability } \\
\hline Feature & Code Type & $\begin{array}{c}\text { Portion of } \\
\text { Patients With } \geq 1 \\
\text { Observations }\end{array}$ & $\begin{array}{c}\text { Average Number of } \\
\text { Observations for Patients } \\
\text { With } \geq 1 \text { Observations }\end{array}$ \\
\hline Evaluation and Management & $\mathrm{CPT}$ & $100 \%$ & 23.03 \\
\hline Pathology & СРT & $96 \%$ & 35.25 \\
\hline Medicine & СРТ & $83 \%$ & 15.11 \\
\hline Other Pancreatic Conditions & DIAG & $82 \%$ & 14.50 \\
\hline Inflammatory Conditions Of Pancreas & DIAG & $81 \%$ & 44.52 \\
\hline Special Encounters & DIAG & $76 \%$ & 34.87 \\
\hline Other Symptoms & DIAG & $76 \%$ & 35.14 \\
\hline Cardiovascular & $\mathrm{CPT}$ & $73 \%$ & 4.35 \\
\hline Ulcer Drugs & NDC & $72 \%$ & 6.03 \\
\hline Radiology & $\mathrm{CPT}$ & $69 \%$ & 6.53 \\
\hline Other Analgesics & NDC & $67 \%$ & 10.23 \\
\hline Symptoms (Abdominal And Pelvis) & DIAG & $65 \%$ & 49.05 \\
\hline Laboratory & REVCODE & $65 \%$ & 23.98 \\
\hline Musculoskeletal & DIAG & $63 \%$ & 23.38 \\
\hline Digestive & DIAG & $58 \%$ & 42.34 \\
\hline Metabolic & DIAG & $58 \%$ & 33.28 \\
\hline Radiology Abdominal & $\mathrm{CPT}$ & $57 \%$ & 4.77 \\
\hline Esophagus & DIAG & $55 \%$ & 23.38 \\
\hline Mental & DIAG & $53 \%$ & 34.26 \\
\hline Antibiotics & NDC & $53 \%$ & 2.25 \\
\hline Other Special Encounters & DIAG & $53 \%$ & 9.54 \\
\hline Respiratory & DIAG & $52 \%$ & 26.16 \\
\hline Pharmacy & REVCODE & $50 \%$ & 9.30 \\
\hline Digestive Surgery & СРТ & $50 \%$ & 4.90 \\
\hline Hypertensive & DIAG & $50 \%$ & 34.33 \\
\hline Anesthesia & СРТ & $49 \%$ & 2.82 \\
\hline Genitourinary & DIAG & $48 \%$ & 26.69 \\
\hline Emergency Room & REVCODE & $47 \%$ & 5.09 \\
\hline Antidepressants & NDC & $45 \%$ & 7.59 \\
\hline Drugs Requiring Specific Identification & REVCODE & $44 \%$ & 8.47 \\
\hline $\begin{array}{l}\text { CPT: current procedural terminology; DI } \\
\text { REVCODE: revenue code } \\
\text { Note: Observations were tabulated at the cla }\end{array}$ & agnosis; EPI: & pancreatic insuffi & y; NDC: national drug code; \\
\hline
\end{tabular}

From our analysis for Model 3 of the impact of each feature on Gini impurity, we found that two features indicating pancreatic diagnoses were the most important determinates in assigning EPI probability. Table 7 summarizes the top 30 features, which accounted for $90 \%$ of the model's decrease in Gini impurity.

Using the Gini impurity approach, we found that among patients with low EPI probability, the absence of diagnosis codes related to conditions of the pancreas and digestive system was the feature that contributed most to low-probability assignments. For example, a randomly selected patient who was assigned a $<10 \%$ probability of EPI did not have any diagnosis codes related to inflammatory conditions 
of the pancreas, other pancreatic conditions, or malabsorption syndromes, and was a younger patient (age 37 years). Conversely, a randomly selected patient who was assigned a $>90 \%$ probability of EPI had been diagnosed with pancreatic conditions and malabsorption syndromes, was an older patient (age 55 years) and had received prescriptions for ulcer drugs.

\section{Table 7. Summary of Model 3 Feature Importance as Measured by Each Feature's Contribution to Decrease in Gini Impurity}

\section{Feature Importance}

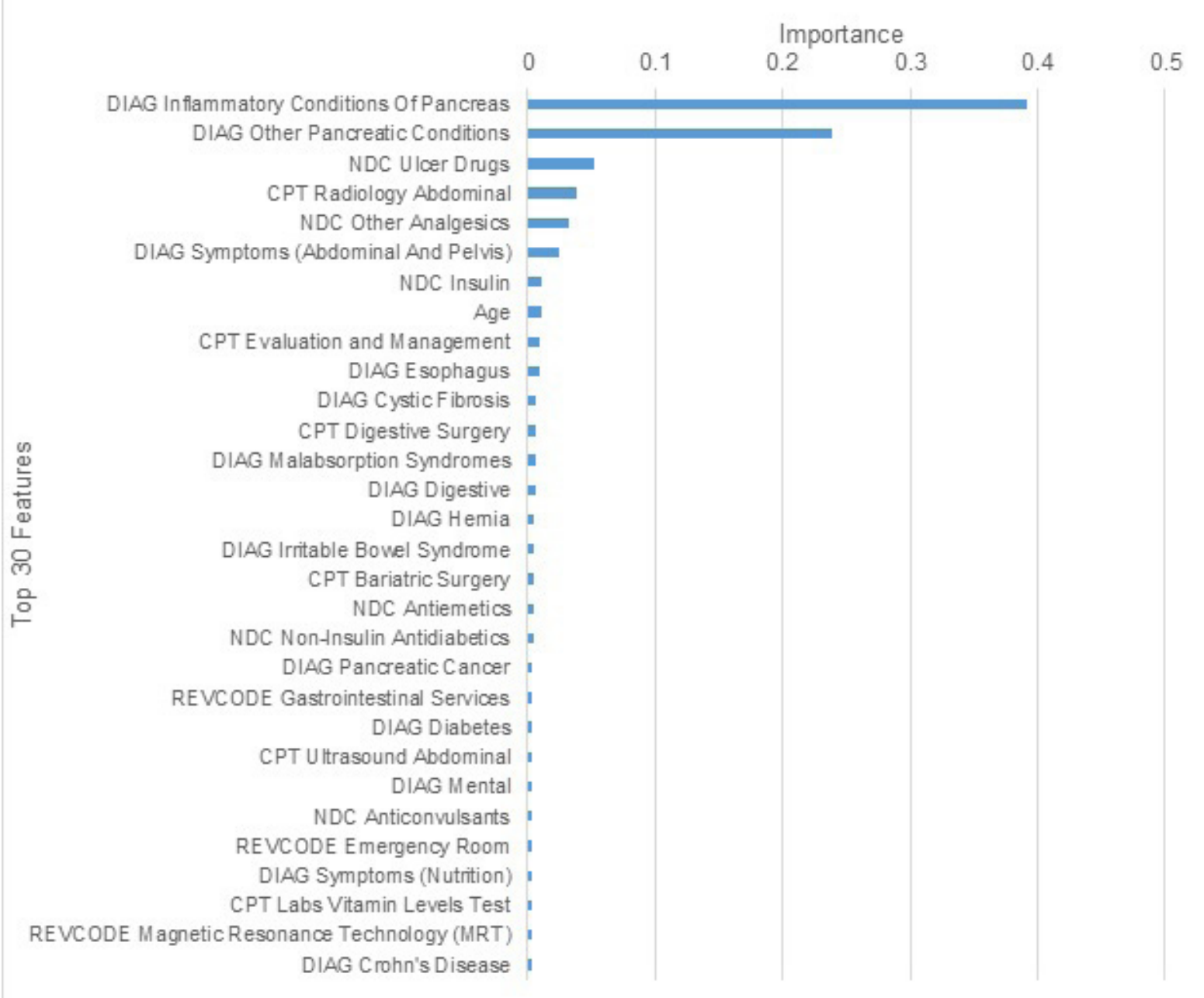

CPT: current procedural terminology; DIAG: diagnosis; NDC: national drug code; REVCODE: revenue code Note: The sum of all features' contributions to the decrease in Gini impurity $=1.00$

\section{Discussion}

EPI is a serious condition that remains underdiagnosed because of the nonspecific nature of clinical symptoms, lack of an ideal diagnostic test, and the inability to easily identify patients with EPI using administrative data. We performed an analysis using machine learning techniques to identify patients in the MarketScan database who likely had EPI but had not been diagnosed with the condition. For the study population, we identified 12 likely EPI patients for every actual EPI patient, a finding that suggests a successful machine learning model for identifying likely undiagnosed patients despite current clinical and administrative barriers. 
Machine learning is still in its infancy, thus studies evaluating its value and applicability have understandably focused mainly on technical methodology, with leaders in the field sharing analytic techniques. In recent years, however, numerous studies have described clinical applications of machine learning that might be accessible to healthcare decision-makers. For example, machine learning techniques have been used to predict a wide range of events and outcomes in medicine, including early hospital readmissions, ${ }^{13}$ laboratory test results, ${ }^{14}$ treatment requirements, ${ }^{21}$ treatment success, ${ }^{15,22}$ disease progression, ${ }^{18}$ disease complications, ${ }^{20}$ posttraumatic stress, ${ }^{19}$ suicide risk, ${ }^{16}$ graft failure after transplantation, ${ }^{17}$ optimal drug dosage, ${ }^{29}$ and adverse drug reactions. ${ }^{24}$ Most notably, the use of machine learning techniques has garnered much attention in several major therapeutic areas, including diabetes, ${ }^{20,30}$ cancer, ${ }^{31}$ cardiology, ${ }^{32}$ ophthalmology, ${ }^{21,33}$ and psychiatry. ${ }^{15,34,35}$ Two recent studies utilized machine learning in particularly novel ways - in one case, to evaluate the trend in sentiment toward papillomavirus vaccination using Twitter data, ${ }^{36}$ and in the other case, to predict swine movements within a regional program to improve the control of infectious diseases in the US. ${ }^{37}$

The use of machine learning offers practical solutions to real-world challenges in healthcare, even those subject to the limitations of real-world data. Our findings showed that from a public health or market perspective, machine learning is a potentially useful tool to estimate the prevalence of EPI, a condition known to be underdiagnosed. Indeed, the case-finding technique used in our study could be applied to other conditions that may be frequently undiagnosed or nearly impossible to identify in administrative claims data. It is important to note that administrative claims data, which are often used in real-world analytics, are readily available on the scale of tens of millions of lives across multiple years and from virtually all sites of care. A challenging future research endeavor would be to validate our model using EMR, a data source that is notoriously difficult to use on a large-scale basis. Although EMR data provide details of patient complaints and diagnostic values, several barriers to its use should be recognized: the data are complex, patient information may be missing due to the use of multiple providers who are not in the same system, and the consolidation and assemblage of information from different systems and multiple sites pose administrative challenges.

Most of the models we tested in our study performed relatively well; however, we anticipate that additional machine learning and statistical methods used in future studies could potentially improve our probability estimates. In fact, recent studies have examined estimating class priors in unlabeled data and improving models being trained primarily on positive and unlabeled data. ${ }^{38,39}$ To further improve the metrics, a greater number of features - beyond the 290 used in our study - could be added to the inputs to refine the current EPI model, or additional populations could be included to profile for variant EPI subpopulations with mild to moderate pancreatic dysfunction (eg, non-insulin-dependent type 2 diabetics); examples include time between procedures, variables from previous years, and impressions of causal or correlated conditions or treatments from clinical experts. Additional validation of the model's predictability could be achieved by following patients for several years and observing who started PERT therapy, or by mining EMR to look for an EPI diagnosis. Finally, an ICD-10 code for EPI, K86.81, was introduced in October 2016, thus the models used in our study could be revisited to use an actual EPI diagnosis code to identify EPI patients, in addition to using treatment with PERT to indicate actual positives for EPI.

We believe that machine learning models can be practical, cost-effective tools for organizations with the necessary resources, which include strong medical claims database capabilities and knowledge of medicine, actuarial science, and statistics. By way of example, our team consisted of about a dozen individuals with diverse backgrounds, including actuarial, statistical, clinical, and healthcare data analytics, all of whom worked substantially less than fulltime and largely completed the analysis within three months.

We acknowledge several limitations in our study. First, although we used the standard approach of randomizing data into training and testing subsets, we could not verify the findings through chart audits. We regard the 
latter as an important future step and envision that an insurer could validate these findings through its case management efforts and further train the model to increase its accuracy. Second, the resources dedicated to running the models - both time and computer resources - could be constraints for organizations trying to apply our approaches. A small insurer, for example, may not have the resources to evaluate or implement the model. Third, the usual limitations of administrative claims data also applied to our study. Claims data, although comprehensive and adequate in many ways, are subject to variation in provider coding practices and inaccuracies. The latter limitation, however, is not unique to our study, but rather reflects the status of realworld data that any organization implementing a case-finding technique would encounter.

\section{Conclusions}

Administrative claims data, although readily available on the scale of tens of millions of lives, lack many of the clinical details that can be found in EMR; however, obtaining large-scale EMR data has proved to be difficult. Machine learning approaches applied to administrative claims data offer a fast and practical approach to researching important healthcare challenges.

The high predictive power of our EPI model shows that applying machine learning techniques to administrative claims data can offer practical and efficient solutions to understanding real-world healthcare challenges. Although definitive claims about the implications of our findings on clinical practice cannot be made, we submit that our study has demonstrated the feasibility and potential value of using machine learning as an efficient strategy for predicting EPI in undiagnosed patients and perhaps will inspire future researchers to improve our probability estimates and extend our findings.

\section{Disclosure}

Funding for this work was provided to Milliman, Inc. by AbbVie Inc.

\section{Acknowledgments}

We would like to thank Kathleen Wildasin for her very helpful editorial support. We would also like to thank Marjorie Schulman, MD; Jason Tsai, MD, MS of AbbVie Inc.; as well as Shea Parkes, FSA; Christine Ferro; Sean Pittinger; Mona Kelkar; and Melissa Caplen of Milliman, Inc. for their technical support. Finally, we would like to thank Rachelle Fox of ICON Plc. for her oversight of the approval process.

\section{References}

1 Alkaade S, Vareedayah AA. A primer on exocrine pancreatic insufficiency, fat malabsorption, and fatty acid abnormalities. Am J Manag Care. 2017;23(12 suppl):S203-9.

2 Fieker A, Philpott J, Armand M. Enzyme replacement therapy for pancreatic insufficiency: present and future. Clin Exper Gastroenterol. 2011;4:55-73.

3 Struyvenberg MR, Martin CR, Freedman SD. Practical guide to exocrine pancreatic insufficiency - breaking the myths. BMC Med. 2017;15:29.

${ }^{4}$ Lindkvist B. Diagnosis and treatment of pancreatic exocrine insufficiency. World J Gastroenterol. 2013;19(42):7258-66.

${ }^{5}$ Löhr J-M, Oliver MR, Frulloni L. Synopsis of recent guidelines on pancreatic exocrine insufficiency. United Eur Gastroenterol J. 2013;1(2):79-83. 
${ }^{6}$ Domínguez-Muñoz JE, Iglesias-García J, Vilariño-Insua M, et al. 13C-mixed triglyceride breath test to assess oral enzyme substitution therapy in patients with chronic pancreatitis. Clin Gastroenterol Hepatol. 2007;5(4):4848.

7 Domínguez-Muñoz JE. Pancreatic enzyme therapy for pancreatic exocrine insufficiency. Gastroenterol Hepatol. 2011;7(6):401-3.

8 Toouli J, Biankin AV, Oliver MR, et al. Management of pancreatic exocrine insufficiency: Australasian Pancreatic Club recommendations. Med J Aust. 2010;193(8):461-7.

9 Frulloni L, Falconi M, Gabbrielli A, et al. Italian consensus guidelines for chronic pancreatitis. Dig Liver Dis. 2010;42(suppl 6):S381-406.

${ }^{10}$ Shafiq N, Rana S, Bhasin D, et al. Pancreatic enzymes for chronic pancreatitis. Cochrane Database Syst Rev. 2009;(4):CD006302.

${ }^{11}$ Sabater L, Ausania F, Bakker OJ, et al. Evidence-based guidelines for the management of exocrine pancreatic insufficiency after pancreatic surgery. Ann Surg. 2016;264(6):949-58.

${ }^{12}$ Leeds JS, Hopper AD, Sidhu R, et al. Some patients with irritable bowel syndrome may have exocrine pancreatic insufficiency. Clin Gastroenterol Hepatol. 2010;8(5):8433-8.

${ }^{13}$ He D, Mathews SC, Kalloo AN, et al. Mining high-dimensional administrative claims data to predict early hospital readmissions. J Am Med Inform Assoc. 2014;21:272-9.

${ }^{14}$ Luo Y, Szolovits P, Dighe AS, et al. Using machine learning to predict laboratory test results. Am J Clin Pathol. 2016;145(6):778-88.

${ }^{15}$ Young J, Kempton MJ, McGuire P. Using machine learning to predict outcomes in psychosis. Lancet Psychiatry. 2016;3(10):908-9.

${ }^{16}$ Cheng Q, Li TM, Kwok CL, et al. Assessing suicide risk and emotional distress in Chinese social media: a text mining and machine learning study. J Med Internet Res. 2017;19(7):e243.

${ }^{17}$ Lau L, Kankanige Y, Rubinstein B, et al. Machine-learning algorithms predict graft failure after liver transplantation. Transplantation. 2017;101(4):e125-32.

${ }^{18} \mathrm{Hu}$ X, Reaven PD, Saremi A, et al. Machine learning to predict rapid progression of carotid atherosclerosis in patients with impaired glucose tolerance. EURASIP J Bioinform Syst Biol. 2016;2016(1):14.

${ }^{19}$ Saxe GN, Ma S, Ren J, et al. Machine learning methods to predict child posttraumatic stress: a proof of concept study. BMC Psychiatry. 2017;17(1):223.

${ }^{20}$ Dagliati A, Marini S, Sacchi L, et al. Machine learning methods to predict diabetes complications. J Diabetes Sci Technol. 2018;12(2):295-302.

${ }^{21}$ Bogunovic H, Waldstein SM, Schlegl T, et al. Prediction of Anti-VEGF treatment requirements in neovascular AMD using a machine learning approach. Invest Ophthalmol Vis Sci. 2017;58(7):3240-8.

${ }^{22}$ Acion L, Kelmansky D, van der Laan M, et al. Use of a machine learning framework to predict substance use disorder treatment success. PLoS One. 2017;12(4):e0175383.

${ }^{23}$ Kang J, Schwartz R, Flickinger J, et al. Machine learning approaches for predicting radiation therapy outcomes: a clinician's perspective. Int J Radiat Oncol Biol Phys. 2015;93(5):1127-35.

${ }^{24}$ Raja K, Patrick M, Elder JT, et al. Machine learning workflow to enhance predictions of adverse drug reactions (ADRs) through drug-gene interactions: application to drugs for cutaneous diseases. Sci Rep. 2017;7(1):3690.

${ }^{25}$ SAS. Machine learning. What it is \& why it matters. https://www.sas.com/en_us/insights/analytics/machinelearning.html. Accessed March 20, 2018. 
${ }^{26}$ Pedregosa F, Varoquau G, Gramfort A, et al. Scikit-learn: machine learning in Python. J Mach Learn Res. 2011;12:2825-30.

${ }^{27}$ Tibshirani R. Regression shrinkage and selection via the lasso. J Royal Stat Soc Ser B. 1994;58:267-88.

${ }^{28}$ Bergstra J, Bengio Y. Random search for hyper-parameter optimization. J Mach Learn Res. 2012;13(1):281-305.

${ }^{29}$ Tang J, Liu R, Zhang YL, et al. Application of machine-learning models to predict tacrolimus stable dose in renal transplant recipients. Sci Rep. 2017;7:42192.

${ }^{30}$ Kavakiotis I, Tsave O, Salifoglou A, et al. Machine learning and data mining methods in diabetes research. Comput Struct Biotechnol J. 2017;15:104-16.

${ }^{31}$ Kourou K, Exarchos TP, Exarchos KP, et al. Machine learning applications in cancer prognosis and prediction. Comput Struct Biotechnol J. 2015;13:8-17.

${ }^{32}$ Forssen H, Patel R, Fitzpatrick N, et al. Evaluation of machine learning methods to predict coronary artery disease using metabolomic data. Stud Health Technol Inform. 2017;235:111-5.

${ }^{33}$ De Fauw J, Keane P, Tomasev N, et al. Automated analysis of retinal imaging using machine learning techniques for computer vision. Version 2. F1000 Res. 2017;5:1573.

${ }^{34}$ Bhagyashree SIR, Nagaraj K, Prince M, et al. Diagnosis of dementia by machine learning methods in epidemiological studies: a pilot exploratory study from South India. Soc Psychiatry Psychiatr Epidemiol. 2018;53(1):77-86.

${ }^{35}$ Sato JR, Biazoli CE Jr, Salum GA, et al. Association between abnormal brain functional connectivity in children and psychopathology: a study based on graph theory and machine learning. World J Biol Psychiatry. 2018;19(2):119-29.

${ }^{36} \mathrm{Du} \mathrm{J}, \mathrm{Xu}$ J, Song HY, et al. Leveraging machine learning-based approaches to assess human papillomavirus vaccination sentiment trends with Twitter data. BMC Med Inform Decis Mak. 2017;17(suppl 2):69.

${ }^{37}$ Valdes-Donoso P, VanderWaal K, Jarvis LS, et al. Using machine learning to predict swine movements within a regional program to improve control of infectious diseases in the US. Front Vet Sci. 2017;4(2):1-13.

${ }^{38} \mathrm{du}$ Plessis MC, Sugiyama M. Class prior estimation from positive and unlabeled data. IEICE Transactions Inform Syst. 2014;E97-D(5):1358-62.

${ }^{39}$ Sakai T, du Plessis MC, Niu G, et al. Semi-supervised classification based on classification from positive and unlabeled data. Version 4. June 16, 2017. http://arxiv.org/abs/1605.06955. Accessed March 20, 2018. 